\title{
MORFOLOGIA DOS ESTÔMATOS EM FOLÍOLOS DE AMENDOIM, CULTIVARES TATU E SO-909 $\left(^{1}\right)$
}

\author{
RENATO FERRAZ DE ARRUDA VEIGA $(2,5)$, GRACI MIRIAN CORSO $(3)$ \\ e PAULO ROBERTO CURI ( $)$
}

\begin{abstract}
RESUMO
Procurando conhecer a variabilidade dos estômatos ocorrentes nos folíolos do amendoim (Arachis hypogaea L.), cultivares Tatu e SO-909, estudaram-se os tipos de estomatos, o índice estomático e dimensőes, com os germoplasmas SO-53 (Tatu') e SO-909 (PI-259747), obtidos do Banco Ativo de Germoplasma đo Instituto Agronômico (IAC). Nas seções paradérmicas dos folíolos, observaram-se quatro tipos básicos de estômatos: anomocítico, anisocítico, diacítico e laterocíclico, além de estômatos geminados. Os laterocíclicos apresentaram-se com maior freqüência. Os diacíticos não são citados na literatura consultada sobre o gênero Arachis. A largura dos estômatos e o índice estomático mostraram diferenças entre os genótipos.
\end{abstract}

Termos de indexação: amendoim, Arachis hypogaea L., morfologia, estômatos.

\section{ABSTRACT \\ STOMATA LEAFLET MORPHOLOGY OF PEANUT, CULTIVARS TATU AND SO-909}

The variability in the stomata of peanut (Arachis hypogaea L.) were studied in two cultivars with different responses to some of the peanut foliar diseases. The results of analysis showed four types of stomata, namely anomocytic, anisocytic, diacytic, and laterocyclic; in addition, the geminate stomata was also found. The highest frequencies were observed for the laterocyclics. The diacytic type has not been cited in the literature, either in the Leguminosaea family or in the genus Arachis. Differences between cultivars as the stomata width and index might produce different peanut cultivar responses to foliar fungal diseases.

Index terms: peanut, Arachis hypogaea L., morphology, stomata.

(1) Trabalho recebido para publicação em 6 de agosto đe 1991 e aceito em 30 de setembro de 1992.

( ${ }^{2}$ Sistema de Introduçāo e Quarentena de Plantas, Instituto Agronômico (IAC), Caixa Postal 28, 13001-970 Campinas (SP).

(3) Departamento de Botânica, Universidade Fstadual Paulista (UNESP), Caixa Postal 178, 13500 Rio Claro (SP). (SP).

4) Serviço de Estatística, Faculdade de Medicina, Veterinária e Zootecnia, Universidade Estadual Paulista (UNESP), 18610 Botucatu

(5) Com bolsa de pesquisa do CNPq. 


\section{INTRODUÇÃO}

Apesar de o Brasil ser considerado o centro de origem e dispersão das espécies do gênero Arachis (Winton, 1904; Hoehne, 1940; Krapovickas, 1968; Valis, 1983; Pietrarelli \& Bonetto, 1986), esse germoplasma tem sido pouco estudado morfologicamente no País.

O uso de dados morfológicos sobre estômatos de amendoim tem despertado interesse, especialmente dos botânicos (Reed, 1924; Yarbrough, 1957), porém muitos dados também são encontrados em trabalhos de fitopatologia (Hemingway, 1957; Abdou, 1966; Mazzani et al., 1972).

O tipo dos estômatos tem sido descrito por vários autores (Fryns-Claessens \& Cotthen, 1973); optou-se, porém, neste trabalho, pelas definiçōes de Wilkinson (1977), por se considerá-las amplas, atuais e abrangentes aos tipos observados no amendoim.

As mediçōes de estômatos e o índice estomático têm sido aplicados também na área de fitopatologia, por se relacionarem com uma das vias de entrada, para determinados fungos, no interior dos folíolos, onde o maior índice estomático, a maior largura e o maior comprimento possibilitam um caminho mais fácil de penetração (Hemingway, 1957; Abdou, 1966).

O presente trabalho teve como objetivos conhecer a variabilidade dos tipos de estômatos existentes nos cultivares Tatu e SO-909 e detectar a importância da sua freqüência e dimensōes sobre as moléstias fúngicas foliares.

\section{MATERIAL E METODOS}

O germoplasma de Arachis hypogaea L. utilizado no presente trabalho foi fornecido pelo Banco Ativo de Germoplasma de amendoim, do Instituto Agronômico (IAC).

Escolheu-se o cultivar Tatu por predominar, em área de plantio, nas regiōes produtoras do Brasil (Lasca, 1984). Como o cultivar escolhido é considerado suscetível à maior das moléstias fúngicas foliares, optou-se pela inclusão do germoplasma SO-909, que possui respostas de resistência opostas ao 'Tatu', à maioria das moléstias foliares (Savy Filho \& Moraes, 1977; Moraes et al., 1978, 1983; Moraes \& Salgado, 1983; Moraes \& Savy Filho, 1983), e por pertencerem à subespécie fastigiata (Waldron, 1919), variedade fastigiata, tipo botânico Valência (Gregory et al., 1951).
O cultivar Tatu (SO-53), cuja origem é desconhecida, provavelmente provenha de sementes selecionadas por fazendeiros paulistas, e o SO-909 (PI-259747) é oriundo do Uruguai.

As excicatas utilizadas encontram-se nos seguintes herbários: CTES, do Instituto de Botânica del Nordeste, em Corrientes (Argentina); BOTU, da UNESP-Botucatu (SP), e IAC, Instituto Agronômico de Campinas (SP).

O local escolhido para o plantio do experimento de campo foi o Centro Experimental de Campinas (IAC), sendo o terreno preparado e adubado de acordo com Godoy et al. (1986).

As sementes foram previamente tratadas com fungicida em pó Rhodiauran e, para evitar interferência dos defensivos químicos na morfologia das plantas, nāo se realizou o controle de pragas e moléstias.

O experimento foi em blocos ao acaso, com seis blocos e dois tratamentos, sendo as parcelas constituídas de três linhas de $2 \mathrm{~m}$, no espaçamento de $0,70 \times 0,20 \mathrm{~m}$; as observaçōes foram feitas na linha central, servindo as laterais de bordadura.

Efetuaram-se as amostragens nos foliolos superiores na quarta folha expandida a partir do ápice do caule, 90 dias após o plantio.

As seçōes paradérmicas foram realizadas no material a fresco, à mão livre, na regiāo mediana do folíolo, no limbo foliolar, entre a nervura central e a marginal. Os cortes foram corados com verde-iodo acético e vermelho-congo (Dop \& Gautié, 1909) e montados em lâminas semipermanentes com gelatina glicerinada.

As seçōes foram fotografadas em fotomicroscópio Nikon, utilizando-se filme Panatomic-x-ASA 32-35 mm.

As medidas foram feitas com auxilio de micrômetro de ocular. Calculou-se a freqüência dos diferentes tipos de estômatos e o número de estômatos por milímetro quadrado, a partir de contagens com ocular de retículo em cem campos, no terço mediano das faces adaxial e abaxial de cinco folhas por germoplasma.

Analisaram-se os tipos estomáticos de acordo com Wilkinson (1977), e calculou-se o índice estomático segundo Salisbury (Metcalfe \& Chalk, 1977), através da seguinte fórmula: 


$$
\mathrm{IE}=\mathrm{E} /(\mathrm{C}+\mathrm{E}), 100
$$

onde:

$$
\text { IE = índice estomático; }
$$

$\mathrm{E}=$ número de estômatos por $\mathrm{mm}^{2}$, e

$\mathrm{C}=$ número de células epidérmicas por $\mathrm{mm}^{2}$.

Para as variáveis medidas, efetuou-se o estudo descritivo, determinando-se, em cada germoplasma: média $(\overline{\mathbf{x}})$, mediana (Md), desvio-padrāo (s), coeficiente de variação $(\mathrm{CV})$, intervalo de confiança da média a $95 \%$, intervalo que inclui $95 \%$ dos valores, percentis (P5 e P95) e estatística (t).

Realizou-se a comparaçāo entre cultivares por intermédio do teste $t$ (Berquó et al., 1980) e, nas demais variáveis, adotaram-se testes näo paramétricos (Siegel, 1975).

Nas comparaçōes entre germoplasmas, empregaram-se os testes de Mann-Whitney (Siegel, 1975).

Para comparação entre faces em cada germoplasma, utilizou-se o teste de Wilcoxon (Siegel, 1975).

Devido ao tipo de variável (contagens em geral), optou-se pela prova não paramétrica de Wilcoxon (Siegel, 1975), em lugar de sua alternativa paramétrica, o teste t.

Utilizou-se a prova em duas situações: (1) para pequenas amostras quando $\mathrm{NC}<25$, onde $\mathrm{NC}$ é o número de pares em que nāo se verificou empate; (2) para grandes amostras, quando $\mathrm{NC}>25$.

A hipótese de nulidade (HO) foi rejeitada quando $\mathrm{p}<0,05$.

\section{RESULTADOS}

Os estômatos são do tipo anomocítico, lateroć́clico, anisocítico e diacítico, sendo o laterocíclico o mais freqüente (Figuras 1, 2 e 3), notando-se a presença de raros estômatos geminados (Figura $3 \mathrm{C}$ ), sendo o tipo anomocítico somente observado, com maior freqüência, no folíolo do 'SO-909' (Figura 2A, Quadros 1 e 2).

Nos folíolos, a freqüência de estômatos do tipo laterocíclico, por milímetro quadrado, na face adaxial do 'Tatu', foi de $132,35 \mathrm{~F} / \mathrm{mm}^{2}$, e no 'SO-909', de $120,78 \mathrm{~mm}^{2}$; na face abaxial, de $117,45 \mathrm{~F} / \mathrm{mm}^{2}$ para o 'Tatu' e de $115,88 \mathrm{~F} / \mathrm{mm}^{2}$ para o 'SO-909' (Quadros 1 e 2).
A freqüência dos estômatos diacíticos, na face adaxial dos folíolos, foi de $0,59 \mathrm{~F} / \mathrm{mm}^{2}$ para o 'Tatu'

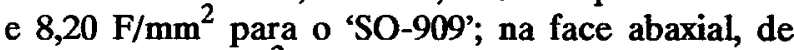
1,96 e $0,98 \mathrm{~F} / \mathrm{mm}^{2}$, respectivamente, para o 'Tatu'e o 'SO-909'.

A freqüência dos estômatos do tipo anisocítico, na face adaxial dos folíolos, foi de $1,37 \mathrm{~F} / \mathrm{mm}^{2}$ para o 'Tatu' e 3,53 F/mm ${ }^{2}$ para o 'SO-909'; na abaxial, de 5,10 e $5,69 \mathrm{~F} / \mathrm{mm}^{2}$, respectivamente, para 'Tatu' e 'SO-909'.

Os anomocíticos não foram registrados nas duas faces dos folíolos do 'Tatu'. O 'SO-909' apresentou, na face adaxial, freqüência de $0,59 \mathrm{~F} / \mathrm{mm}^{2}$, e, na abaxial, de 1,57 F/mm ${ }^{2}$.

A freqüência de estômatos geminados na face adaxial do folíolo do 'Tatu' foi de $0,20 \mathrm{~F} / \mathrm{mm}^{2}$, e no 'SO-909', de $0,39 \mathrm{~F} / \mathrm{mm}^{2}$; na face abaxial, não foi registrada para o 'SO-909', sendo de $0,20 \mathrm{~F} / \mathrm{mm}^{2}$ no 'Tatu'.

O índice estomático, na face adaxial dos folíolos, foi de 16,50 e 15,73 e, na abaxial, de 15,08 e 12,33, respectivamente, para o 'Tatu' e para o 'SO-909'.

Os estômatos dos folíolos, na face adaxial do 'Tatu' medem, em média, 27,90 $\mu \mathrm{m}$ de comprimento por $22,24 \mu \mathrm{m}$ de largura; os do 'SO-909', 28,00 por $20,10 \mu \mathrm{m}$. Na face abaxial, o 'Tatu' mede 33,10 $\mu \mathrm{m}$ de comprimento por $25,87 \mu \mathrm{m}$ de largura, e o 'SO-909', 30,76 $\mu \mathrm{m}$ por 20,76 $\mu \mathrm{m}$ (Quadros 3, 4 e 5).

Anomocítico
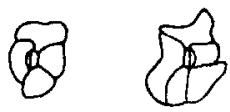

Laterociclico
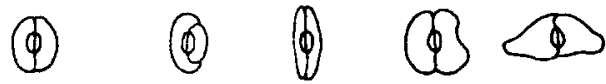

Anisocítico
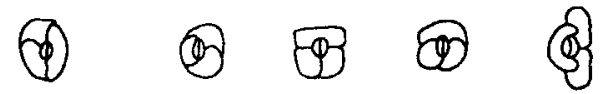

Diacítico
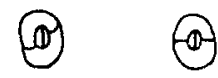

Figura 1. Tipos de estômatos, em desenhos fora de escala, observados em folíolos de Arachis hypogaea L., cultivares Tatu e SO-909. 
Quadro 1. Freqüência de estômatos e índice estomático em ambas as faces dos folíolos de Arachis hypogaea L., cultivares Tatu e SO-909

Estrutura

Epiderme adaxial

'Tatu' 'SO-909'

Epiderme abaxial

'Tatu'

'SO-909 $\mathrm{F} / \mathrm{mm}^{2}$

Estômatos laterocíclicos

Estômatos diacíticos

Estômatos anisocíticos

Estômatos anomocíticos

Estômatos geminados

Total de estômatos

Total de células epidérmicas

Índice estomático

\begin{tabular}{rrrr} 
& & $\mathrm{F} / \mathrm{mm}^{2}$ & \\
\cline { 3 - 4 } 132,35 & 120,78 & 117,45 & 115,88 \\
0,59 & 8,20 & 1,96 & 0,98 \\
1,37 & 3,53 & 5,10 & 5,69 \\
$\ldots$ & 0,59 & $\ldots$ & 1,57 \\
0,20 & 0,39 & 0,20 & $\ldots$ \\
134,31 & 125,10 & 124,51 & 124,12 \\
679,80 & 670,39 & 701,18 & 882,74 \\
16,50 & 15,73 & 15,08 & 12,33 \\
\hline
\end{tabular}

...: não encontrado.

Quadro 2. Valores da mediana (Md) e do posto médio (R) para cada genótipo de amendoim 'Tatu' e 'SO-909'.

Estatística z na aproximação normal do teste de Mann-Whitney e nível de significância

\begin{tabular}{|c|c|c|c|c|}
\hline Variável & Valores & 'Tatu' & 'SO-909' & Estatística \\
\hline $\begin{array}{l}\text { Estômatos tipo anomocítico, } \\
\text { epiderme face abaxial, } \mathbf{n}^{\mathrm{Q}}\end{array}$ & $\begin{array}{r}\mathbf{M d} \\
\mathbf{R}\end{array}$ & $\begin{array}{c}0 \\
96,5\end{array}$ & $\begin{array}{c}0 \\
104,5\end{array}$ & $\begin{array}{l}z=2,88 \\
p=0,04\end{array}$ \\
\hline $\begin{array}{l}\text { Estômatos tipo anomocítico, } \\
\text { epiderme face adaxial, } \mathbf{n}^{\mathrm{Q}}\end{array}$ & $\begin{array}{r}\mathbf{M d} \\
\mathbf{R}\end{array}$ & $\begin{array}{c}0 \\
99,0\end{array}$ & $\begin{array}{c}0 \\
102,0\end{array}$ & $\begin{array}{l}z=1,74 \\
p=0,08\end{array}$ \\
\hline $\begin{array}{l}\text { Estômatos geminados, } \\
\text { epiderme face abaxial, } \mathbf{n}^{\mathbf{o}}\end{array}$ & $\begin{array}{r}\mathbf{M d} \\
\mathbf{R}\end{array}$ & $\begin{array}{c}0 \\
101,0\end{array}$ & $\begin{array}{c}0 \\
100,0\end{array}$ & $\begin{array}{l}z=1,00 \\
p=0,32\end{array}$ \\
\hline $\begin{array}{l}\text { Estômatos geminados, } \\
\text { epiderme face adaxial, } \mathbf{n}^{\circ}\end{array}$ & $\begin{array}{r}\mathbf{M d} \\
\mathbf{R}\end{array}$ & $\begin{array}{c}0 \\
100,0\end{array}$ & $\begin{array}{c}0 \\
101,0\end{array}$ & $\begin{array}{l}z=0,58 \\
p=0,56\end{array}$ \\
\hline $\begin{array}{l}\text { Células epidérmicas, } \\
\text { face abaxial, } \mathbf{n}^{\mathbf{2}}\end{array}$ & $\begin{array}{r}\text { Md } \\
\mathbf{R}\end{array}$ & $\begin{array}{l}35 \\
82,63\end{array}$ & $\begin{array}{c}38 \\
118,37\end{array}$ & $\begin{array}{l}z=4,38 \\
p<0,001\end{array}$ \\
\hline $\begin{array}{l}\text { Células epidérmicas, } \\
\text { face adaxial, } \mathbf{n}^{\mathrm{Q}}\end{array}$ & $\begin{array}{r}\text { Md } \\
\mathbf{R}\end{array}$ & $\begin{array}{c}34 \\
105,07\end{array}$ & $\begin{array}{l}34 \\
95,93\end{array}$ & $\begin{array}{l}z=1,12 \\
p=0,26\end{array}$ \\
\hline
\end{tabular}

Os valores apresentados referem-se à área do retículo de ocular. 

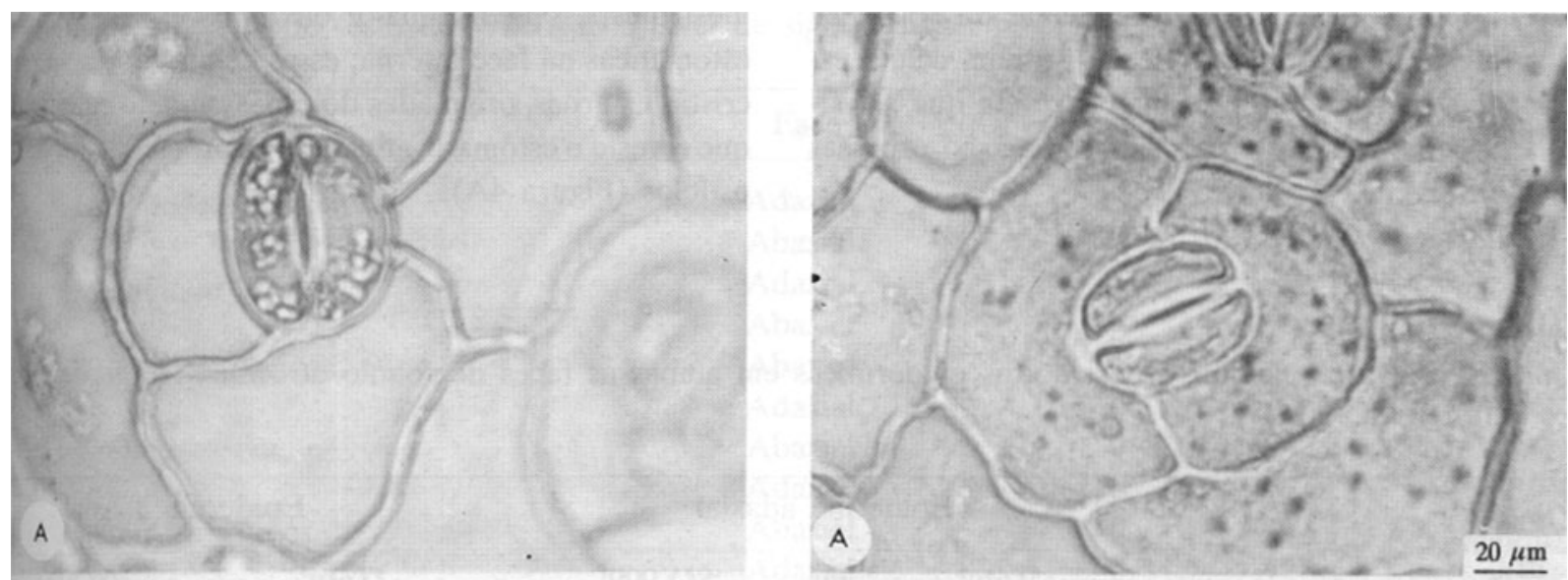

B

$20 \mu \mathrm{m}$
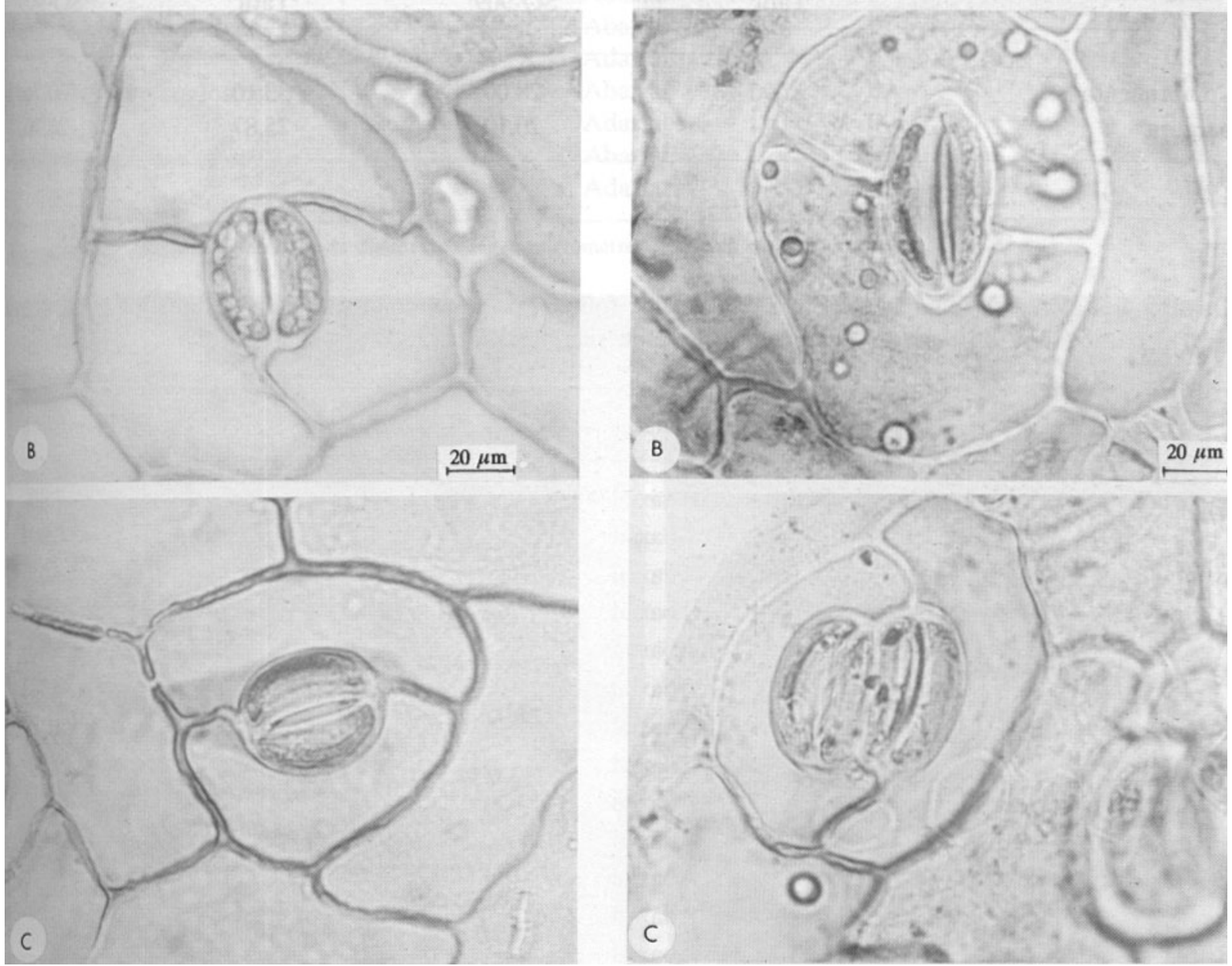

Figura 2. Seçāo paradérmica de folíolo de Arachis hypogaea L. 'Tatu'. Tipos de estômatos: A: anomocítico; B: anisocítico; C: laterocíclico.

Figura 3. Seção paradérmica de folíolo de Arachis hypogaea L. 'Tatu'. Tipos de estômatos: A: diacítico; B: diacítico; C: geminados. 
As células estomáticas, em seção transversal, estão no mesmo nível das demais células da epiderme dos folíolos (Figura 4). A seção de uma célula estomática, segundo seu eixo maior, revela que as paredes periclinais externa e interna são espessas (Figura 4B).
A seção transversal mediana dos estômatos mostra espessamento maior das paredes das células estomáticas na face interna; essas células apresentam cristas externas, originadas do espessamento cuticular que reveste o estômato, atingindo as proximidades dos ostíolos (Figura 4A).

Quadro 3. Valores médios de estruturas epidérmicas em ambas as faces do folíolo de Arachis hypogaea L., 'Tatu' e 'SO-909'

Estômato

Epiderme adaxial

'Tatu' 'SO-909'

Epiderme abaxial

'Tatu'

'SO-909’

$\mu \mathrm{m}$

28,00

33,10

25,87

30,76

20,10

Quadro 4. Valores da comparação entre faces dos folíolos do amendoim cv. Tatu. Estatística z na prova não paramétrica do teste de Wilcoxon e nível de significância

\begin{tabular}{|c|c|c|c|}
\hline Variável & Face & Mediana & Estatîstica \\
\hline \multirow[t]{2}{*}{ Estômato: comprimento, $\mu \mathrm{m}$} & Adaxial & 8 & $z=7,80$ \\
\hline & Abaxial & 10 & $p<0,001$ \\
\hline \multirow[t]{2}{*}{ Estômato: largura, $\mu \mathrm{m}$} & Adaxial & 7 & $z=8,05$ \\
\hline & Abaxial & 8 & $p<0,001$ \\
\hline \multirow[t]{2}{*}{ Estômatos laterocíclicos, $\mathrm{n}^{\mathrm{Q}}$} & Abaxial & 6 & $z=3,40$ \\
\hline & Adaxial & 7 & $p<0,001$ \\
\hline \multirow[t]{2}{*}{ Estômatos diacíticos, $\mathbf{n}^{\mathcal{Q}}$} & Abaxial & 0 & $t=21$ \\
\hline & Adaxial & 0 & $p>0,10$ \\
\hline \multirow[t]{2}{*}{ Estômatos anisocíticos, $\mathrm{n}^{\mathrm{Q}}$} & Abaxial & 0 & $z=2,85$ \\
\hline & Adaxial & 0 & $p=0,004$ \\
\hline \multirow[t]{2}{*}{ Estômatos anomocíticos, $\mathbf{n}^{\mathrm{o}}$} & Abaxial & 0 & $\ldots$ \\
\hline & Adaxial & 0 & $\ldots$ \\
\hline \multirow[t]{2}{*}{ Estômatos geminados, $\mathbf{n}^{\mathbf{o}}$} & Abaxial & 0 & $\mathrm{t}=1,5$ \\
\hline & Adaxial & 0 & $p>0,20$ \\
\hline \multirow[t]{2}{*}{ Células epidérmicas, $\mathbf{n}^{\mathfrak{Q}}$} & Abaxial & 35 & $z=2,36$ \\
\hline & Adaxial & 34 & $p=0,018$ \\
\hline
\end{tabular}

Os valores referem-se às divisões da régua micrométrica e à área do retículo de ocular. ...: não encontrados. 
Quadro 5. Valores da comparação entre faces dos folíolos do amendoim cv. SO-909. Estatística z na prova não paramétrica do teste de Wilcoxon e nível de significância

\begin{tabular}{|c|c|c|c|}
\hline Variável & Face & Mediana & Estatística \\
\hline \multirow[t]{2}{*}{ Estômato, comprimento, $\mu \mathrm{m}$} & Adaxial & 8 & $z=6,58$ \\
\hline & Abaxial & 9 & $\mathrm{p}<0,001$ \\
\hline \multirow[t]{2}{*}{ Estômato, largura, $\mu \mathrm{m}$} & Adaxial & 6 & $z=2,15$ \\
\hline & Abaxial & 6 & $p=0,032$ \\
\hline \multirow[t]{2}{*}{ Estômatos laterocíclicos, $\mathrm{n}^{\mathbf{9}}$} & Abaxial & 6 & $z=1,14$ \\
\hline & Adaxial & 6 & $p=0,26$ \\
\hline \multirow[t]{2}{*}{ Estômatos diacíticos, $\mathbf{n}^{\mathbf{2}}$} & Abaxial & 0 & $t=3,5$ \\
\hline & Adaxial & 0 & $p>0,10$ \\
\hline \multirow[t]{2}{*}{ Estômatos anisocíticos, $\mathbf{n}^{\mathbf{Q}}$} & Abaxial & 0 & $z=1,40$ \\
\hline & Adaxial & 0 & $p=0,16$ \\
\hline \multirow[t]{2}{*}{ Estômatos anomocíticos, $\mathrm{n}^{\mathrm{e}}$} & Abaxial & 0 & $\mathrm{t}=31,5$ \\
\hline & Adaxial & 0 & $p>0,10$ \\
\hline \multirow[t]{2}{*}{ Estômatos geminados, $\mathrm{n}^{\mathrm{Q}}$} & Abaxial & 0 & $t=0$ \\
\hline & Adaxial & 0 & $\mathbf{p}=1$ \\
\hline \multirow[t]{2}{*}{ Células epidérmicas, $\mathbf{n}^{\mathrm{Q}}$} & Abaxial & 38 & $z=6,24$ \\
\hline & Adaxial & 24 & $p<0,001$ \\
\hline
\end{tabular}

Os valores apresentados referem-se às đivisỏes da régua micrométrica e à área do retículo ocular.
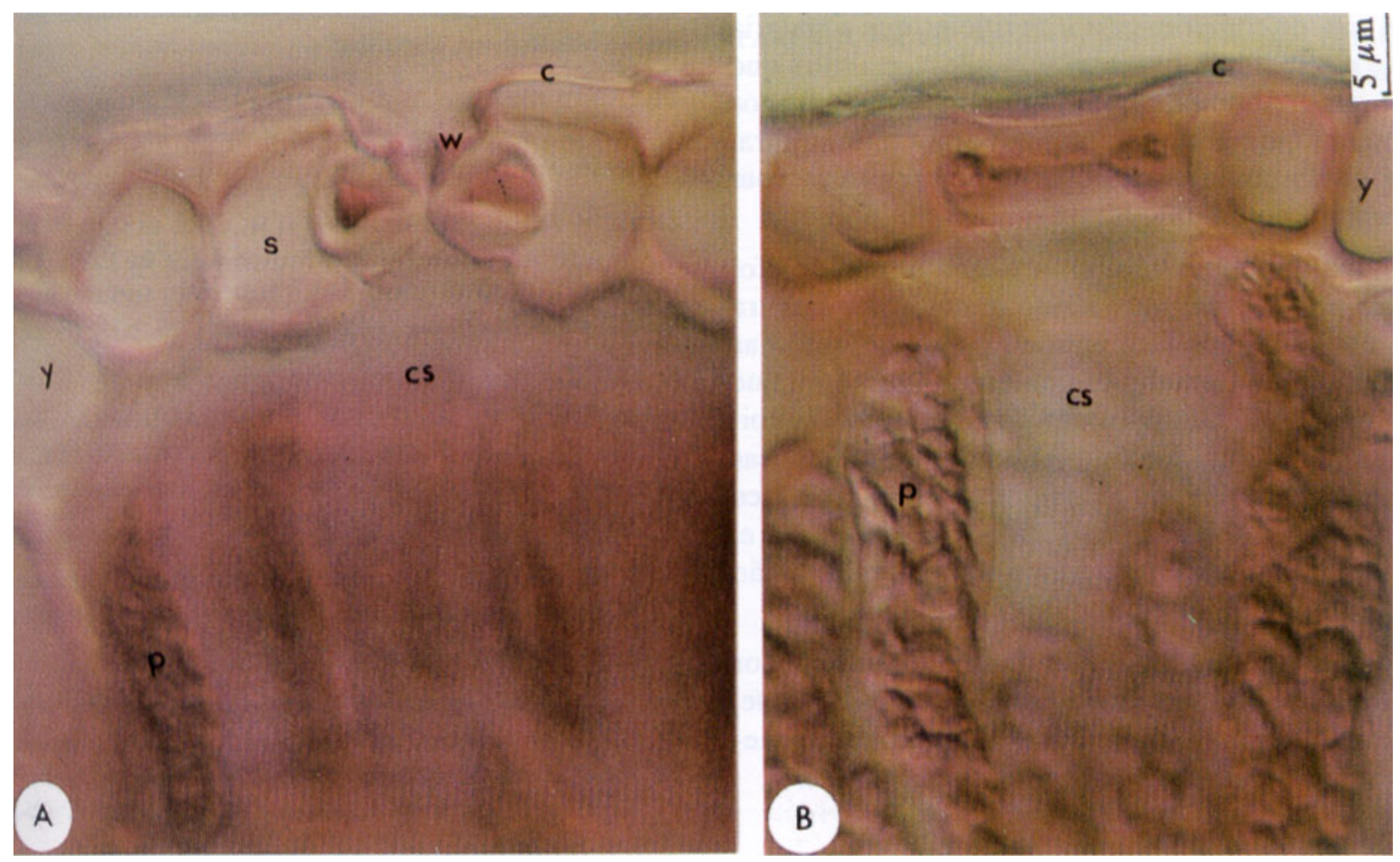

Figura 4. Pormenores de estômatos em seção transversal do folíolo de Arachis hypogaea L. A: 'Tatu', face adaxial. Seçăo na região međiana do estômato: crista (w); cutícula (c); células subsidiárias (s); câmara subestomática (cs); parênquima paliçádico (p); células epidérmicas (y). B: 'Tatu', face adaxial. Seção segundo o maior eixo da célula estomática: cutícula (c); câmara subestomática ( $c s)$; parênquima paliçádico ( $p$ ); células epidérmicas $(\mathrm{y})$. 


\section{DISCUSSÃO}

Não foram encontrados registros pormenorizados sobre estômatos no gênero Arachis, apenas citaçōes de Yarbrough (1957) de que as células estomáticas variam na sua posição, podendo estar no mesmo plano ou abaixo, em depressão na superfície.

No presente trabalho, as células estomáticas dos dois genótipos aparecem no mesmo nível das demais células epidérmicas.

Nos trabalhos consultados, observou-se coincidência de citações de presença dos estômatos dos tipos laterocíclico e anomocítico; na realidade, observou-se que existem outros tipos, como anisocítico e diacítico.

As mediçōes dos estômatos foram efetuadas por Hemingway (1957) e Mazzani et al. (1972), com o intuito de correlacioná-las com a maior ou menor penetraçāo de fungos. Mazzani et al. (1972) não observaram diferenças na penetração, tanto nos menores, em comprimento, que $14 \mu \mathrm{m}$, como nos maiores que $16 \mu \mathrm{m}$. Hemingway (1957) não encontrou nenhuma penetração nos menores que $13,4 \mu \mathrm{m}$ de comprimento e verificou que a maioria dos estômatos penetrados são maiores que $16,7 \mu \mathrm{m}$. Abdou (1966) observou que os fungos penetram em estômatos com mais de $2 \mu \mathrm{m}$ de largura, não encontrando correlação de comprimento com a penetração do fungo.

No presente trabalho, o índice estomático foi maior no 'Tatu', o que, aliado a sua maior largura dos estômatos, na face abaxial, pode proporcionar aos fungos um caminho de penetração mais fácil que no 'SO-909'. O comprimento dos estômatos da epiderme da face adaxial não mostrou diferenças significativas entre os genótipos, porém, na face abaxial, os estômatos do 'Tatu' são mais longos e, portanto, mais suscetíveis a uma eventual penetração do fungo.

A largura dos estômatos da fase abaxial é maior do que a da adaxial nos dois genótipos, o que, hipoteticamente, permite maior facilidade de penetração de fungos nos estômatos daquela face.

\section{CONCLUSÕES}

A maior resistência a determinadas moléstias fúngicas foliares, mostrada pelo 'SO-909', pode estar supostamente ligada ao menor índice estomático e à maior largura do estômato, no caso da moderada resistência apresentada a Cercosporidium personatum (pinta-preta), na fase de penetração. Para as demais moléstias foliares, não foi possível correlacionar esses dados.

Nos dois cultivares, os estômatos do tipo laterocíclico são os mais comuns, enquanto os dos tipos anisocítico e diacítico são menos freqüentes. Apesar de ocorrer em menor freqüência, o anomocítico é uma citaçāo inédita para o gênero Arachis.

\section{REFERÊNCIAS BIBLIOGRÁFICAS}

ABDOU, Y.A.M. The sources and nature of resistance in Arachis L. species to Mycosphaerella arachidicola Jenk and Mycosphaerella berkeleyii Jenk, and factors infuencing sporulation of these fungi. Raleigh, 1966. 118p. Tese (Doutorado) North Carolina University, 1966.

BERQUÓ, E.S.; SOUZA, I.M.P. \& GOTLIEB, L.D. Bioestatística. São Paulo, Pedadógica e Universitária, 1980. 325p.

DOP, P. \& GAUTIÉ, A. Manual de technique botanique. Paris, F.R de Rudeval, 1909. 534p.

FRYNS-CLAESSENS, E. \& COTTHEM, W.V. A new classification of the ontogenetic types of stomata. Botanical Review, Lancaster, 39(1):71-138, 1973.

GODOY, I.J. de; RODRIGUES FILHO, F.S. de O. \& GERIN, M.A.N. Amendoim (Arachis hypogaea L.). In: INSTTTUTO AGRONOMICO (Campinas). Instruçóes agrícolas para o Estado de Sáo Paulo. 3.ed. Campinas, 1986. p.23. (Boletim, 200)

GREGORY, W.C.; SMITH, B.W. \& YARBROUGH, J.A. Morphology, genetics and breeding. In: NATIONAL FERTILIZER ASSOCIATION. The peanut: the umpredictable legume. Washington, 1951. p.28-88.

HEMINGWAY, J.S. The resistance of groundnuts to Cercospora leafspots. Empire Journal of Experimental Agriculture, Oxford, 25:60-68, 1957.

HOEHNE, F.C. Leguminosas-Papilionadas: gênero Arachis precedido da chave geral para as subfamílias, tribos e gêneros das leguminosas do Brasil. In: — . Flora brasilica. Sāo Paulo, Secretaria da Agricultura, Indústria e Comércio, 1940. v.25-II, fasc.2, p.1-20.

KRAPOVICKAS, A. Origen, variabilidad y difusión del mani (Arachis hypogaea). In: CONGRESSO INTERNACIONAL DE AMERICANISTAS, 37., 1968. Actas \& memorias. Buenos Aires, 1968. v.2, p.517-534. 
LASCA, D.H. de C. Amendoim: produçăo em São Paulo. A Granja, Porto Alegre, 40(443):74-78, 1984.

MAZZANI, B.; ALLIEVI, J. \& BRAVO, P. Relación entre la incidencia de manchas foliares por Cercospora spp. y algunas características varietales del mani. Agronomia Tropical, Macaray, 22(2):119-132, 1972.

METCALFE, C.R. \& CHALK, L. Anatomy of the dicotyledons. 2.ed. Oxford, Clarendon Press, 1977. v.1.

MORAES, S.A. de; GODOY, I.J. de \& GERIN, M.A.N. Avaliaçāo da resistência de Arachis hypogaea à Puccinia arachidis, Sphaceloma arachidis e Phoma arachidicola. Fitopatologia Brasileira, Brasilia, 8(3):499-506, 1983.

MORAES, S.A. de \& SALGADO, C.L. Reaçôes de seis cultivares de amendoim (Arachis hypogaea) à Cercospora arachidicola e C. personata em folhas destacadas. Fitopatologia Brasileira, Brasilia, 8(2):291-303, 1983.

MORAES, S.A. de \& SAVY FILHO, A. Reaçöés de seis cultivares de amendoim (Arachis hypogaea L.) à Puccinia arachidis Speg. Summa Phytopathologica, Piracicaba, 9(1/2):140-153, 1983.

MORAES, S.A. de; SOAVE, J. \& SAVY FILHO, A. Reaçōes de 10 variedades de amendoim (Arachis hypogaea $L$.) à verrugose (Sphaceloma arachidis Bit. \& Jenk.). Ecossistema, Espírito Santo do Pinhal, 3(3):43-46, 1978.

PIETRARELLI, J.R. \& BONETTO, L.A. coords. Mani, historia, importancia, técnica de cultivo, uso y comercialización. Manfredi, Instituto Nacional de Técnologia Agropecuária - Estación Experimental Agropecuária Manfredi, 1986. 52p. (Cuaderno de actualización, 3)
REED, E.L. Anatomy, embriology and ecology of Arachis hypogaea. Botanical Gazette, Chicago, 78(3):289-310, 1924.

SAVY FILHO, A. \& MORAES, S.A. de. Observaçōes sobre a incidência de cercosporiose em cultivares de amendoim (Arachis hypogaea L.). Revista de Agricultura, Piracicaba, 52(1):39-46, 1977.

SIEGEL, S. Estatística não paramétrica. Săo Paulo, McGraw-Hill, 1975. 350p.

VALLS, J.F.M. Collection of Arachis germplasm in Brazil. Plant Genetic Resources Newsletter, Roma, (53):9-14, 1983.

WALDRON, RA. The peanut (Arachis hypogaea): its history, histology and utility. Contributions from the Botanical Laboratory of the University of Pennsylvania, Philadelphia, 4:301$-308,1919$.

WILKINSON, H.P. The plant surface (mainly leaf): Part I. Stoma. In: METCALFE, C.R. \& CHALK, L. Anatomy of the dicotyledons. 2.ed. Oxford, Clarendon Press, 1977. v.1, p.97-117.

WINTON, A.L. The anatomy of the peanut with special reference to its microscopic identification in food products. Annual Report of the Connecticut Agricultural Experiment Station, New Haven, 28:191-198, 1904.

YARBROUGH, J.A. Arachis hypogaea: the seedling, its epicotyl and foliar organs. American Journal of Botany, Baltimore, 44(1):19-30, 1957. 